\title{
Scattering Transform using Randomized Averages
}

\author{
Preethika Nandakumar \\ M.Tech. Student, Dept. Computer Science and \\ Engineering \\ Amrita Vishwa Vidyapeetham \\ Coimbatore, India
}

\author{
K A Narayanankutty \\ Professor, Dept. Electronics and Communication \\ Engineering \\ Amrita Vishwa Vidyapeetham \\ Coimbatore, India
}

\begin{abstract}
The purpose of this work aims at implementation of scattering transform using random projection based averages which could result in better high frequency reconstruction and computation speed. First Scattering transform is applied on the image and first order coefficients are extracted. For computing the second order coefficients random projection averaged values of the first order values are used to compute next level transform. The results show that the edge quality of the image is enhanced and run-time of the method got reduced.
\end{abstract}

\section{General Terms}

Image Processing, Wavelet

\section{Keywords}

Scattering Transform, Randomized Averages, Wavelets, Compressed Sensing, Projections.

\section{INTRODUCTION}

On the transmitter side, image is compressed in order to reduce data transmission and in the process of compression [1], [2] some less significant frequencies are discarded. The transmitted low and high frequencies are used during the decompressing process. This results in distortion while reconstructing the image at the receiving end. And to recover this lost information many methods and filters have been proposed in both spatial and frequency domains. In frequency domain wavelet- based coding is widely used in the compression technique. Wavelets use orthonormal basis functions which are simple, its transform results in localized values both in frequency and time domain. Biorthogonal Wavelets [4], [5] used are introduced by Daubechies which contain biorthogonal basis functions. Wavelet transform decompose spectrum into frequency bands on the lower frequency side. These methods fail when frequency of the image goes higher than the window function. Hence it reveals only local features of an image i.e., only small details can be observed. When the frequency goes higher it loses much information. In order to retain this information scattering transform [3] was introduced. However, the averaging phenomenon requires a relook as this may introduce a smoothening effect on the image.

\section{SCATTERING TRANSFORM}

A scattering transform has the structure of convolutional network but its filter is replaced by wavelets. It is an extension of the multi-resolution analysis as proposed by Mallat [3].

By applying wavelet operator [6] [7] on an image signal, using two functions namely the scaling function $(\phi)$ and wavelet function $(\psi)$ all the low frequencies are present in scaling and high frequencies are present in wavelet coefficients. Visually perceivable information is mostly represented by the low frequency components. For transmission only these low frequency components are used which will result in a comparatively less quality reconstructed image. For better results scattering transform [8] [9] [10], is used where it recovers the lost information by averaging with a cascade of wavelet decompositions and using the modulus operator recursively:

$$
S_{,} x(t)=x * \phi_{,}(t)
$$

Here, signal is convolved with scaling function which results in low frequency components and all the high frequency components are removed. In order to retain high frequency component, modulus operator is applied on first wavelet function.

$$
S_{j} x(t)=\left|x * \psi_{j 1}\right|
$$

By this operation only the high frequency components are obtained. To get both high frequencies and as well as low frequencies, averaging on modulus operator of first wavelet function $\left(\psi_{1}\right)$ and convolution with scaling function $(\phi)$ is performed.

$$
S_{J} x(t)=\left|x * \psi_{j 1}\right| * \phi_{J}(t)
$$

The above equation represents first order scattering coefficients.

$$
S_{J} x(t)=\| x * \psi_{j 1}\left|* \psi_{j 2}\right| * \phi_{J}(t)
$$

This co-efficient is again used on modulus operator on second wavelet function and averaged scaling function, resulting in second order scattering coefficients.

Thus $S_{J} x(t)$ contains scattering coefficients, for calculating relations between the original signal $(x)$ and the successive $\psi_{1}, \psi_{2}, \ldots \psi_{\mathrm{g}}$. A scattering transform totals all these coefficients up to a maximum order $q \leq m$.

For any path $p=\left(j_{1}, j_{2} \ldots j_{m}\right)$ of order $m$

$$
S[p] x(t)=|| x * \psi_{j 1}\left|* \psi_{j 2}\right| .\left|* \psi_{j m}\right| * \phi_{j}(t)
$$




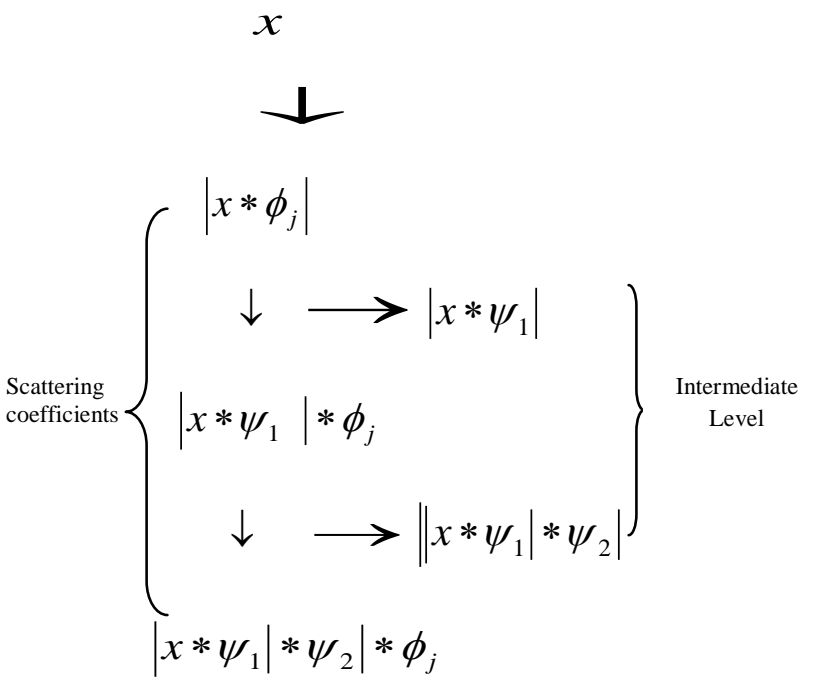

Fig 1 Architecture Diagram of Scattering Transform

\subsection{Inverse Scattering Transform}

Since scattering transform [11] is performed by cascade of wavelet modulus operation, the inversion approximately inverts each layer by layer. At the maximum order $m$, the algorithm begins with a de-convolution estimating the intermediate level but certain details cannot be reconstructed exactly due to subsampling of the signal. Thus de-convolution operation fails. Hence de-convolution is computed using Richardson-Lucy algorithm [12], which preserves the information if $\phi \geq 0 . y_{0}$ is introduced for interpolating the scattering coefficients linearly on the sampling of $x$. The Richardson-Lucy de-convolution iteratively computes,

$$
y_{n+1}=y_{n}(t) \cdot\left[\left(\frac{y_{0}}{y_{n} * \phi}\right) * \tilde{\phi}(t)\right]
$$

Where $\tilde{\phi}(t)=\phi(-t)$. Once calculation of intermediate level is done by using de-convolution, signal $\mathrm{x}$ can be restored by each layer using the advantage of the wavelet coefficients which defines a complete and redundant signal representation. If $\left|x * \psi_{j 1}\right|=0$ then no phase needs to be reconstructed, inversion fails. However since the main source of errors are produced in the de-convolution stage, fast inversion algorithm is used though it gives an approximate result. Griffin \& Lim [13] showed that an alternating projection algorithm can be used to recover the signal exactly, but with large mean square errors because the algorithm is trapped in local minima.

When $m=1$, an approximation $x$ is computed by first estimating the intermediate level using the scattering coefficients with the Richardson Lucy de-convolution algorithm and followed by Griffin \& Lim algorithm for inverting the coefficients.

When $m=2$, an approximation $\bar{x}$ is calculated from scattering coefficients by estimating intermediate levels using de-convolution algorithm and successively inverting with the by Griffin \& Lim algorithm.

\section{PRELUDE TO THE NEW METHOD}

There are three main parts to the brain, cerebrum, cerebral and stem where the cerebral hemisphere is divided into right hemisphere and left hemisphere. The left hemisphere is analytical and logical which helps us to remember facts to deal with abstract information. The right hemisphere puts meaning to those facts which is random and global to make the information to be specific. By considering the working of the human brain, processing of brain can be divided into sequential and random processing. In sequential processing (left - side brain), brain processes the information in linear manner i.e., it processes from part to whole. It takes pieces, lines them up, and arranges them in a logical order; then it draws conclusions. On the other hand random processing (right - hand side) processes from whole to part, which will result in increased processing speed of the information. Randomization of information is analogous to the working of right hemisphere brain.

Compressed sensing [14], is a new method of generating samples based on random projections of the signal, which enables us to recover it with far less samples as that required by the sampling theorem. However the recovery involves more computations. It combines the benefits of sparsity in the frequency domain, properties of the random matrices and information theory. Random projections seem to introduce a deterministic component in the signal for recovery. To increase the computational speed and perception level of an image, randomized projections for the averages are performed on the scattering coefficients.

\subsection{Randomized - Average Scattering Transform}

As discussed, randomization of information is analogous to the working of right hemisphere brain. It makes the computation process faster. In this method, Scattering coefficients are randomized by using small projections over the signal which preserves the structure of the signal and the signal is then reconstructed from these projections. On applying scattering transform on the image first layer scattering coefficients are obtained. These coefficients are convolved with random projections i.e., before averaging with the second wavelet function random values are convolved upon this value and by applying scattering transform second layer coefficients are obtained. These are reconstructed using inverse scattering transform and output is compared with scattering transform.

\subsection{Experimental Results}

The proposed method gives better results by enhancing the features, and is presented here.

a) Original Image
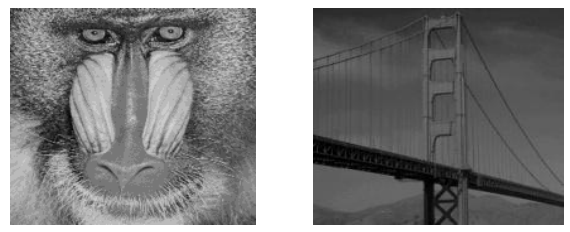
b) After Randomized Scattering Transform
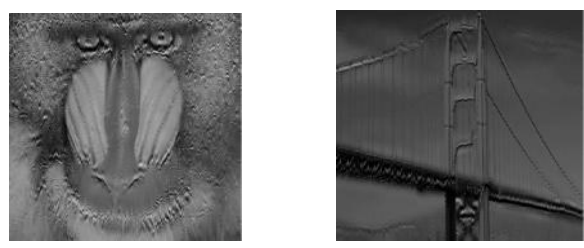

c)

Intensity Corrected Image
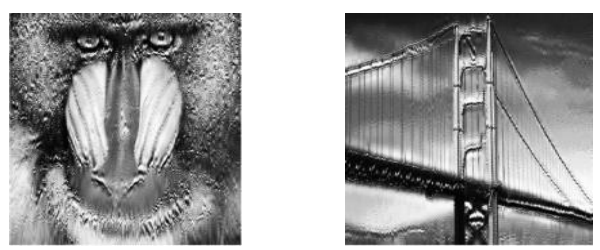

Fig 2 Output of Proposed Method

d)
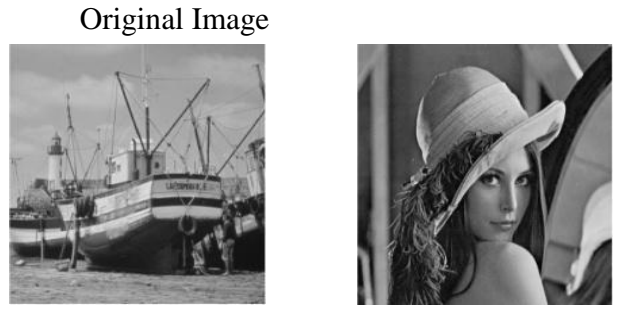

e) After Scattering Transform
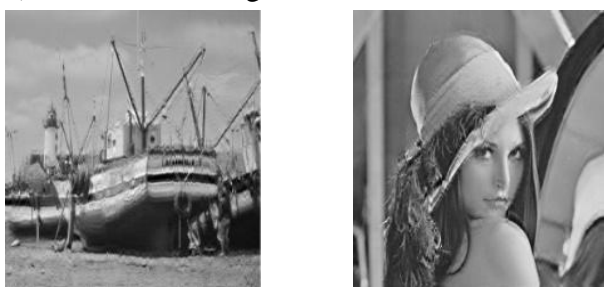

f) Intensity Corrected Image
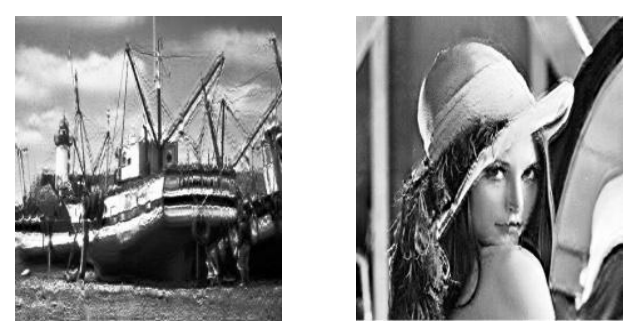

Fig 3 Output of Normal Scattering Transform g) After randomized Scattering Transform
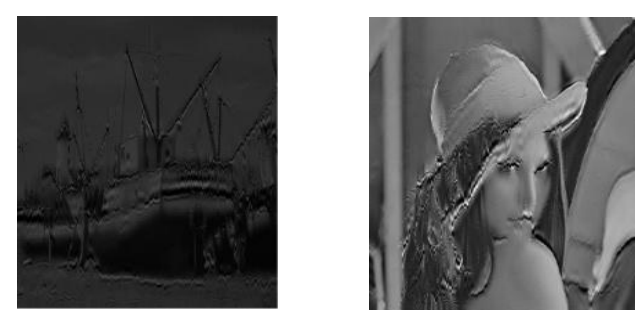

h) Intensity Corrected Image
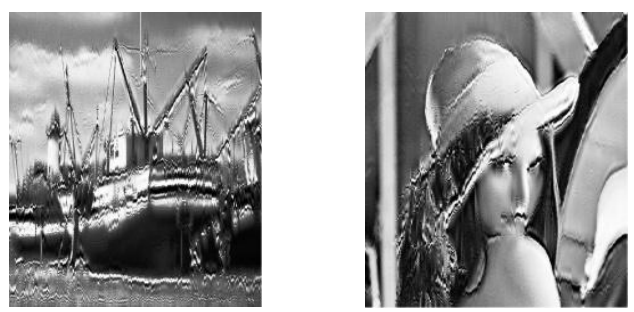

Fig 4 Ouput of Scattering Trasform using Random Averages.
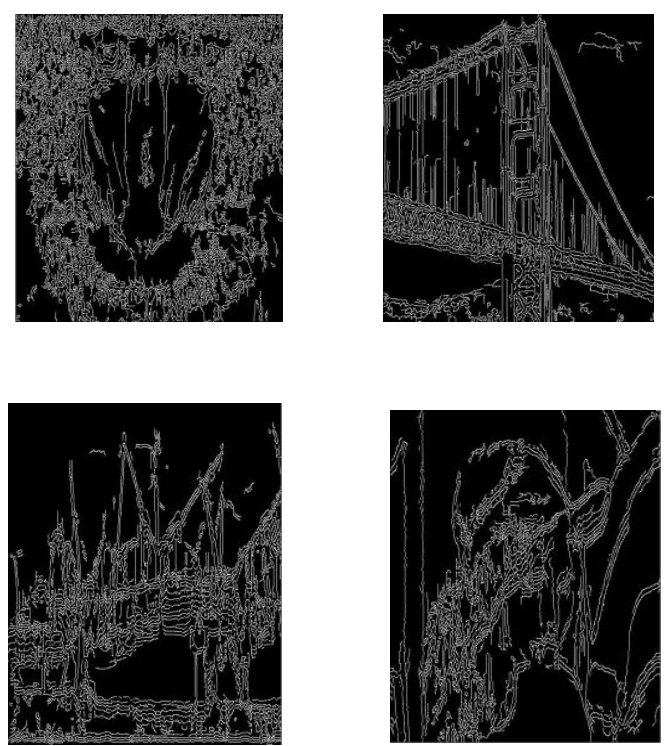

Fig 5 Edges Detected after Normal Scattering Transform 

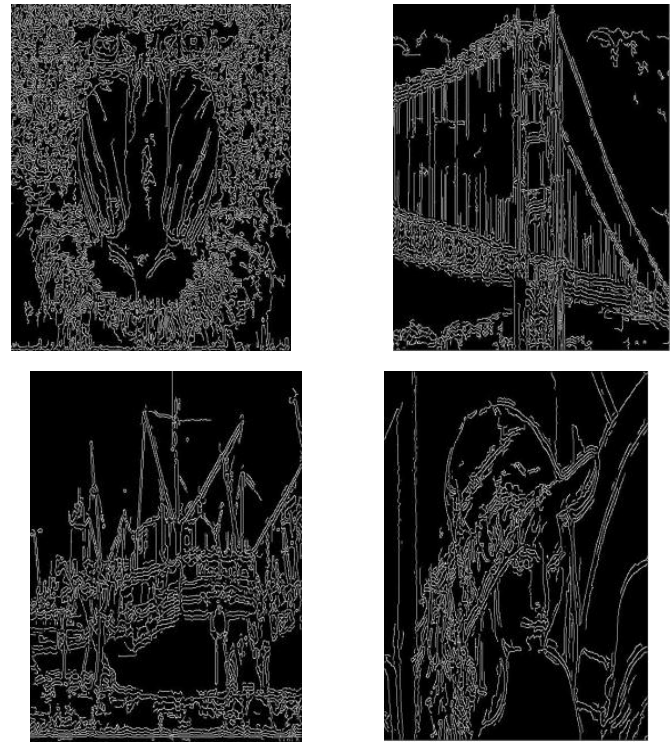

Fig 6 Edges Detected after Randomized Scattering Transform

From the above images it can be infered that the randomized scattering transform detects the edges more effectively than the normal scattering transform.

\subsection{Comparison of Methods}

The proposed method is compared with scattering transform, which yields better result both in perception level and computation time. For perception level, subjective evaluation is performed with the input image and resulted in an expected result. Peak Signal Noise Ratio (PSNR) values are calculated. Since the edges are enhanced PSNR value of the randomized average scattering transform goes less that of the scattering transform.

Table 1 Comparison of PSNR Value

\begin{tabular}{|c|c|c|c|}
\hline S.NO & Dataset & $\begin{array}{c}\text { Normal } \\
\text { Scattering } \\
\text { Transform }\end{array}$ & $\begin{array}{c}\text { Randomized } \\
\text { Average Scattering } \\
\text { Transform }\end{array}$ \\
\hline 1 & Image 1 & 68.9485 & 62.5115 \\
\hline 2 & Image 2 & 73.4067 & 63.5781 \\
\hline 3 & Image 3 & 76.1155 & 62.5459 \\
\hline
\end{tabular}

By comparing the computation time of both the methods, proposed method yields less processing time.

Table 2 Comparison of Runtime

\begin{tabular}{|c|c|c|c|}
\hline S.NO & Dataset & $\begin{array}{c}\text { Normal } \\
\text { Scattering } \\
\text { Transform }\end{array}$ & $\begin{array}{c}\text { Randomized } \\
\text { Average Scattering } \\
\text { Transform }\end{array}$ \\
\hline 1 & Image 1 & 668.83 Seconds & 426.45 Seconds \\
\hline 2 & Image 2 & 716.45 Seconds & 502.35 Seconds \\
\hline 3 & Image 3 & 623.45 Seconds & 402.53 Seconds \\
\hline
\end{tabular}

\section{CONCLUSION}

It can be observed that the edges are better enhanced in randomized scattering transform and thus the image quality is improved. And also computation speed is increased dually. Edge enhancement does affect PSNR values adversely, which was expected. It is now essential to investigate further on this subject.

\section{REFERENCES}

[1] Ricardo L. De Queiroz," Processing Jpeg-Compressed Images And Documents", Ieee Transactions On Image Processing, Vol. 7, No. 12, December 1998.

[2] Jin Li, "Image Compression - the Mechanics of the JPEG 2000", Microsoft Research, Signal Processing, One Microsoft Way, Bld. 113/3374, Redmond, WA 98052.

[3] Joakim andén, Stéphane Mallat, Deep Scattering Spectrum, Submitted to IEEE Transcations on Signal Processing, last revised 10 Jan 2014.

[4] Hae-Sung Lee, Yongbum Cho, Hyeran Byun, and Jisang Yoo"An Image Enhancement Technique Based on Wavelets”, BMCV 2000, LNCS 1811, pp. 286-296, 2000, Springer-Verlag Berlin Heidelberg 2000.

[5] S. Mallat, A wavelet tour of signal processing. Academic Press, 1999.

[6] I. Daubechies, Ten Lectures on Wavelets (SIAM, Philadelphia, 1992).

[7] Tutorial on Wavelets for Compressing Images, chapter in "Explosion of Mathematics", SMF 2007. pdf

[8] Bruna J. and Mallat S , Audio texture synthesis with scattering moments, arXiv preprint arXiv:1311.0407, 2013.

[9] Joan Bruna and St'ephane Mallat, Classification with Scattering Operators, Computer Vision and Pattern Recognition (CVPR), 2011 IEEE Conference on, pp. 1561-1566, 2011.

[10] Stéphane Mallat, Group Invariant Scattering, Communications on Pure and Applied Mathematics, Vol. LXV, 1331-1398 (2012).

[11] I. Waldspurger and S. Mallat, "Recovering the phase of a complex wavelet transform," CMAP, Ecole Polytechnique, Tech. Rep., 2012.

[12] L. Lucy, "An iterative technique for the rectification of observed distributions," Astron. J., vol. 79, p. 745, 1974.

[13] D. W. Gri_n and J. S. Lim, "Signal estimation from modified short-time fourier transform," IEEE Trans. Acoust., Speech, Signal Process., vol. 32, no. 2, pp. $236\{243,1984\}$.

[14] K. A. Narayanankutty and K. P. Soman, Understanding Theory Behind Compressed Sensing, International Journal on Sensing, Computing \& Control Vol. 1, No. 2, pp. 80-91, 2011.

[15] J. Bruna and S. Mallat, Invariant scattering convolution network," IEEE Trans. Pattern Anal. Machince. Intelligent., to appear, http://arxiv.org/abs/1203.1513 